\title{
Evolution Mechanism, Monitoring, and Early Warning Method of Water Inrush in Deep-Buried Long Tunnel
}

\author{
Wenfeng Tu $\mathbb{D}$, Liping Li $\mathbb{D}^{\text {, }}$, Shuai Cheng, Diyang Chen, Yongcai Yuan, and Yanhao Chen \\ Geotechnical and Structural Engineering Research Center, Shandong University, Jinan, Shandong 250061, China \\ Correspondence should be addressed to Liping Li; yuliyangfan@163.com
}

Received 22 April 2021; Accepted 11 August 2021; Published 25 August 2021

Academic Editor: Yi Xue

Copyright (C) 2021 Wenfeng Tu et al. This is an open access article distributed under the Creative Commons Attribution License, which permits unrestricted use, distribution, and reproduction in any medium, provided the original work is properly cited.

\begin{abstract}
Water inrush is a serious disaster in tunnel construction. Because the process of water inrush is complicated and there is no effective monitoring and early warning method, the control of water inrush disaster is passive. Firstly, the failure mode and determination method of safety thickness of the antioutburst structure are summarized. The key breakthrough directions and development trends of water inrush mechanism was analyzed. Secondly, the sensitivity ranking and the adjunct characteristics of different types of precursor information are determined by the model test. An improved scheme of microseismic monitoring technology is proposed to locate the spatial position of the water inrush channel, and a multi-information time-space integrated monitoring method for an antioutburst structure is established. By analyzing the evolution law of multi-information in the process of water inrush, the evolution model and its identification method are put forward. Finally, the level of water inrush was determined through the analysis of the dynamic and static reserves of disaster source, and a multilevel and comprehensive warning system of tunnel water inrush is established. Through the remote real-time monitoring and early warning platform and its engineering application, the early warning of water inrush time trend and the early warning of water inrush rupture space position are realized.
\end{abstract}

\section{Introduction}

In recent years, with the development of infrastructure construction in China, a large number of tunnels were built in western mountainous areas. There are some difficulties in the construction of the tunnel, such as large buried depth, long tunnel line, high stress, strong karst, high water pressure, and frequent disasters [1]. Some serious geological disasters often occur during the construction of deep-buried tunnels $[2,3]$, which have the characteristics of high concealment, strong suddenness, and strong destructiveness. There have been many cases of casualties, equipment damage, and delays in construction due to water inrush of tunnels, as shown in Table $1[4,5]$.

With the large-scale construction of karst tunnels, many researchers carry on the study about the prevention and control techniques of water inrush $[6,7]$. The occurrence and evolution of water inrush is a process of coupling force field and seepage field. For the progressive failure of waterresisting rock mass or the infiltration and instability of filling medium, researchers put forward the failure mode of the antioutburst structure using fracture mechanics and hydraulics, and proposed the mechanical model and criteria of water inrush $[8,9]$. Some researchers analyzed the mechanism and evolution process of the seepage instability of filling [10-13]. The calculation method of the safe thickness of the antioutburst structure was established [14-17]. Numerical simulation is an important method to study water inrush $[18,19]$. At present, the commonly used analysis software mainly includes FLAC-3D, RFPA, COMSOL PFC-3D, and PD, etc., and the research content includes seepage-stress coupling of rock mass, pore water pressure characteristics of mining rock mass, and stability of waterbearing faults and surrounding rocks [20-25]. However, due to limitations in software development, it is difficult to consider the complex construction geological environment of tunnel engineering. To verify the theory and make up for the inability of a mechanical model, researchers carried out some water inrush model tests, because of the unfavorable geological environment, the high osmotic pressure, 
TABLE 1: Statistics on water inrush and mud disasters in tunnels.

\begin{tabular}{|c|c|c|c|}
\hline Tunnel type & Tunnel name & Time/year & Disaster description \\
\hline \multirow{12}{*}{ Railway tunnel } & Yuanliangshan tunnel & 2002 & $\begin{array}{l}\text { Large-scale water and mud inrush occurred } 71 \text { times. The highest water pressure } \\
\text { reached } 4.6 \mathrm{MPa} \text {. The maximum water inflow was } 72,000 \mathrm{~m}^{3} / \mathrm{h} \text {, causing } 9 \text { deaths }\end{array}$ \\
\hline & Maluqing tunnel & 2005 & A large-scale water and mud inrush occurred 19 times, causing 11 deaths \\
\hline & Yesanguan tunnel & 2007 & $\begin{array}{l}\text { In a half-hour, water irruption quantity is } 151,000 \mathrm{~m}^{3} \text {, and mudstone eruption } \\
\text { quantity is } 53,500 \mathrm{~m}^{3} \text {, causing } 10 \text { deaths }\end{array}$ \\
\hline & Guzishan tunnel & 2011 & A major water inrush occurred and the surface collapsed, causing 2 deaths \\
\hline & Taoshuping tunnel & 2014 & Water inrush, causing 9 deaths \\
\hline & Wulong tunnel & 2002 & $\begin{array}{l}\text { There have been more than } 10 \text { large-scale water and mud inrush, with a maximum } \\
\text { water inflow of } 7.18 \text { million } \mathrm{m}^{3} / \mathrm{d}\end{array}$ \\
\hline & Yunwushan tunnel & 2008 & The water inflow is $46,000 \mathrm{~m}^{3} / \mathrm{d}$, and the maximum water inflow is $172,000 \mathrm{~m}^{3} / \mathrm{d}$ \\
\hline & Qiyueshan tunnel & 2005 & $\begin{array}{c}\text { Exposing } 187 \text { large-scale karst pipes and caves, and causing large-scale water inrush } \\
\text { for more than } 20 \text { times }\end{array}$ \\
\hline & Dazhiping tunnel & 2006 & $\begin{array}{l}\text { The water and mud inrush reaches more than } 60,000 \mathrm{~m}^{3} \text { and then stabilizes } \\
\text { at } 8000 \mathrm{~m}^{3}\end{array}$ \\
\hline & Xiangshan tunnel & 2009 & $\begin{array}{c}\text { The maximum water inflow reaches } 7300 \mathrm{~m}^{3} / \mathrm{h} \text {, and multiple water inrush occurs } \\
\text { within two years. }\end{array}$ \\
\hline & Bahuashan tunnel & 2006 & $\begin{array}{l}\text { The total water inflow is } 15,000 \mathrm{~m}^{3} \text {, and the protruding angle crushed stone is about } \\
1800 \mathrm{~m}^{3} \text {. There are } 13 \text { sudden water and mud inrush accidents before and after, and } \\
\text { the } 200 \mathrm{~m} \text { long tunnel is inundated. }\end{array}$ \\
\hline & Chaoyang tunnel & 2018 & $\begin{array}{l}\text { The maximum water inflow was } 1100 \mathrm{~m}^{3} / \mathrm{h} \text {, and the water output was estimated to } \\
\text { be about } 100,000 \mathrm{~m}^{3} \text { causing } 3 \text { deaths }\end{array}$ \\
\hline \multirow{6}{*}{ Road tunnel } & Zhongjiashan tunnel & 2012 & $\begin{array}{l}\text { The water and mud inrush occurred } 14 \text { times in } 3 \text { months, the total mud volume } \\
\text { exceeded } 27,900 \mathrm{~m}^{3} \text {, and the total water inrush exceeded } 20,000 \mathrm{~m}^{3}\end{array}$ \\
\hline & Longtan tunnel & 2006 & $\begin{array}{l}\text { Large-scale burst mud } 2 \text { times; the mud volume exceeded } 9000 \mathrm{~m}^{3} \text {, causing } \\
\text { large-scale landslide } 3 \text { times }\end{array}$ \\
\hline & Baiyunshan tunnel & 2010 & $\begin{array}{l}\text { Large-scale instant burst mud } 2000 \mathrm{~m}^{3} \text {, maximum water inflow } 300 \mathrm{~m}^{3} / \mathrm{h} \text {, surface } \\
\text { subsidence } 300 \mathrm{~m}^{2} \text {, depth } 20 \mathrm{~m}, 6 \text { deaths }\end{array}$ \\
\hline & Foling tunnel & 2012 & The inflow of water is $30,000 \mathrm{~m}^{3}$, and the pumping water is 1.3 million cubic meters. \\
\hline & Shangjiawan tunnel & 2013 & $\begin{array}{l}\text { Three large-scale water inrush occurred in the same section, and the maximum } \\
\text { amount of inrush of clay and water suddenly reached } 7700 \mathrm{~m}^{3} \text {. }\end{array}$ \\
\hline & Anshi tunnel & 2019 & $\begin{array}{l}\text { About } 15,000 \mathrm{~m}^{3} \text { of mud and } 800 \mathrm{~m}^{3} \text { of water gushed into the disaster site causing } \\
12 \text { deaths. Ten people were injured. }\end{array}$ \\
\hline
\end{tabular}

complex stress, and construction disturbance encountered during the construction process [26]. The test system of a similar physical model was used to study the seepage and deformation laws of surrounding rock under the combination of multiple factors, which provided a theoretical basis for the antiseepage reinforcement technology during the construction process [27-32].

Damage and destruction of the antioutburst structure will lead to the occurrence of water inrush. The damage process will be accompanied by various physical information (stress, strain, acoustic emission, etc.). Rock failure test is the basis to obtain the evolution law of multiple information. It is also an important basis for the monitoring design of the precursory information of the antioutburst structure [33-41]. Nevertheless, most of the basic mechanical tests use single information to explain the damage process of rock mass, and the influence of multiphysics coupling is not considered. Many researchers have developed similar material for the fluid-solid coupling model test [42]. Based on the different formation and experimental conditions, researchers found out the developmental characteristics of fissures under different stress and seepage pressures. In the real-time monitoring of geological disasters, the network application system was developed based on the internet of things, which was successfully applied in the instability monitoring of slope stability, mine impact pressure, and water inrush [43-48]. However, the monitoring method of water inrush mainly relies on geological precursor information and hydrogeological report. In the early warning of water inrush, D-S evidence theory, extension theory, and fuzzy evaluation [49-53] are applied to the fusion analysis of multi-information. The severity of the disasters induced by the disaster-causing structure was graded. This method determines the monitoring items such as water pressure, water quantity and quality, initial support, and surrounding rock stability [54]. Nevertheless, the correlation between precursor information and the water inrush model is not clear, and it is difficult to carry out the research work of early warning for a specific water inrush model. 
Due to the complexity, suddenness, and diversity of the water inrush disasters in deep tunnels, it is necessary to summarize the different cases of water inrush disasters, analyze the types of different water inrush disasters, and study the mechanism of water inrush under different failure modes and the evolution law of precursor multiple information. Data mining is used to establish an information fusion recognition model to provide a basis for water inrush pattern recognition and fusion warning. Based on the analysis of multi-information fusion of water inrush, a monitoring and early warning system for water inrush disasters is established to realize early warning of water inflow, water inrush time, and location, which are effective measures to prevent and control sudden water disasters. Based on systematically summarizing the mechanism of sudden flooding, the research of this paper focuses on the multi-information fusion analysis and prediction and early warning of water inrush, and divides the water inrush hazard level by dynamic and static water quantity assessment. Through multiinformation monitoring combined with microseismic monitoring technology positioning to carry out time-space warning, and achieve effective active prevention and control of major water inrush disasters, it can provide some reference and support for monitoring and warning of tunnel water inrush disaster.

\section{Catastrophic Evolutionary Mechanism of Water Inrush}

The formation of water inrush disasters of a tunnel is affected by many factors. According to different division principles, water inrush disasters are divided into various types. From the perspective of disaster sources, water inrush disasters are divided into crevice-type water inrush, faulttype water inrush, dissolution-cavity-type water inrush, and pipeline and underground dark river water inrush. Considering the different forms of disasters, water inrush disasters are divided into instantaneous water inrush, stable water inrush, and seasonal water inrush. According to the formation form of the water inrush channel, water inrush disasters are divided into geological defect type and nongeological defect type [10]. The evolution process of the water inrush channel is not only related to the properties of the channel but also closely related to external disturbance factors such as water pressure, ground stress, and blasting disturbance.

2.1. The Failure Mode of Antioutburst Structure. Based on the failure mode of the antioutburst structure, the water inrush type is divided into the progressive failure of the resistance structure and filling medium infiltration and instability. The progressive failure of the resistance structure is mainly caused by dynamic disturbance and hydraulic cleavage under high osmotic pressure. The instability of the filling structure can be divided into local osmotic instability and overall slip instability.

2.1.1. The Progressive Failure of Resistance Structure. Based on the division of the failure mode of the antioutburst structure, combined with the theory of fracture mechanics, structural mechanics, and rock mass dynamics, some mechanical models and water inrush criteria were established, such as tensile shear failure and compression shear failure models [55]. The discriminating conditions of the water inrush were obtained. The karst fissure water is prominently defined as the accumulative stage and the unstable stage, and the mechanical mechanism of the long-term interaction of water and rock before water inrush is studied. It is suitable for analyzing the control effect of karst water and water pressure on the stability of surrounding rock [56].

2.1.2. Filling Medium Infiltration and Instability. For filling faulty geological structures such as large cracks, faults, and karst pipes, the formation of water inrush channels is caused by seepage and catastrophic changes of internal filling media, such as fault activation and karst pipeline filling infiltration. From the permeability characteristics of the filling, the filling medium infiltration and instability can be divided into the permeation instability of the filling medium and slip instability of a filling belt [10].

(1) Permeability Instability of Filling Medium. When the filling geological structure is under strong osmotic pressure and excavation, the internal filling medium is continuously eroded which may lead to piping and soil flow. When the filling material is quickly flushed out, it leads to the formation of water inrush channels and causing the seepage instability of the filling medium [57].

(2) Slip Instability of Filling Belt. When there is a large-scale disaster source nearby and the filling geological structure is relatively weak or impervious, the filling structure has the dual characteristics of water blocking and water filling. This type of filling structure is relatively stable and has high water-blocking properties. When the filler is dense, heterogeneous structure with poor water permeability, or impermeable, the entire structure of the filling does not have the conditions for the formation of a potential water inrush channel. Sliding instability of filling belt is easy to occur under the action of strong seepage.

2.2. Minimum Antiburst Safety Thickness. By analyzing the failure mode of the anti-outburst structure and the evolution process of the catastrophe, the calculation method of the minimum antioutburst safety thickness of the tunnel is summarized. The water inrush from the karst tunnel face is caused by the dual action. On the one hand, the tunnel blasting excavation causes the intrinsic crack activation and expansion of the rock mass. On the other hand, under the continuous action of high karst water pressure, the rock mass is softened. Corrosion changes the effective stress between the cracks in the rock mass, causing the crack to expand and penetrate.

Different scholars have proposed some calculation models of antioutburst safety thickness for different engineering geological conditions. The tunnel floor is simplified as a cantilever beam for mechanical analysis using elastic theory. According to its structural integrity, it is divided into 
"complete rock formation" and "incomplete rock formation," and the calculation formula of the safe thickness of the tunnel floor is obtained [58]. Using fracture mechanics and hydraulics theory to analyze the hysteresis effect and multipath effect of tunnel water inrush, a safe thickness calculation model of rock wall based on critical water pressure is established [9]. Based on the analysis of system potential energy control parameters and catastrophic evolution path, a folding catastrophe model is proposed and the minimum safe thickness calculation formula for water inrush is established [59]. The existence of a safe thickness zone increases the difficulty of fracture propagation in hydraulic fracturing. As the excavation progresses, the relaxation zone gradually extends to the safe thickness zone, and the energy threshold of the hydraulic fracturing of the rock mass under the action of the high head is getting lower and lower. When the tunnel face is located within the minimum safe thickness range, the rock mass undergoes splitting and water inrush. The "three districts" theory holds that the area between the tunnel face and water-bearing body can be divided into three parts: the excavation disturbance zone in front of the face, the fracture zone around the water-bearing body, and the intact rock mass protection zone [55]. The water inrush is a dynamic process in which the excavation disturbs the fissure zone and the permeable fissure zone gradually increase, which is the progressive failure process of the antioutburst structure, and is shown as follows:

$$
h_{s} \geq h_{1}+\frac{1}{\pi}\left(\frac{k_{I c}}{1.12 p_{w}}\right)^{2}+h_{3}
$$

where $h_{s}$ is the area of rock protection thickness; $h_{1}$ is the slack thickness zone caused by construction; $h_{3}$ is a fractured zone, which is determined by geophysical exploration and drilling methods; $k_{\mathrm{Ic}}$ is the fracture toughness of rock; $p_{w}$ is fissure water pressure.

In view of the water inrush caused by dynamic disturbance such as blasting excavation, the antioutburst structure of the karst tunnel is divided into blasting excavation disturbance zone $L_{c}$ and hydraulic fracturing zone $L_{w}$ [56]. In engineering practice, the blasting excavation disturbance zone $L_{c}$ can be obtained by a field test, empirical estimation, and theoretical calculation. Hydraulic fracturing zone $L_{w}$ under the action of the explosive stress wave is derived by analyzing the critical water pressure of the expansion and failure of water-bearing cracks in the tunnel face, as shown in equation (2) and equation (3). The formula for calculating the minimum safe thickness of the fractured rock mass is $L=L_{c}+L_{w}$.

$$
\begin{aligned}
& L_{w}=\frac{11 R}{17} \cdot\left\{\ln \lambda-\ln \left[\lambda-\frac{2 f P_{w} \sqrt{\pi a}-\sqrt{3} K \prod^{C}-2 c \sqrt{\pi a}+2 \tau_{1}\left|K_{\prod}^{(2)}\right| \sqrt{\pi a}}{\gamma H \sqrt{\pi a}(f-f \cos 2 \beta-\sin 2 \beta)}\right]-\frac{f+f \cos 2 \beta+\sin 2 \beta}{f-f \cos 2 \beta-\sin 2 \beta}\right\}, \\
& L_{w}=\frac{11 R}{17} \cdot\left\{\ln \lambda-\ln \left[\lambda-\frac{2 f P_{w} \sqrt{\pi a}-\sqrt{3} K \prod^{C}-2 c \sqrt{\pi a}+2 \tau_{1}\left|K_{\prod}^{(2)}\right| \sqrt{\pi a}}{\gamma H \sqrt{\pi a}(f-f \cos 2 \beta+\sin 2 \beta)}\right]-\frac{f+f \cos 2 \beta-\sin 2 \beta}{f-f \cos 2 \beta+\sin 2 \beta}\right\} .
\end{aligned}
$$

2.3. Key Issues of Water Inrush Mechanism Research. The existing theoretical model has certain applicable conditions. For example, in view of the existence of karst caves at the tunnel floor, the beam-slab model simplifies the blast disturbance to the additional force on the rock mass, and hydrostatic pressure is treated as water pressure. The natural rock mass may not only break water in the intact rock mass but also break in the structural plane of the rock mass. Therefore, the criterion of water inrush for the progressive failure of resistance structure established by the theory of fracture mechanics is generally applicable to the brittle failure of rock mass which immediately changes from elastic deformation to sudden fracture. The applicability of this method is poor for large plastic deformation and failure of rock mass after elastic deformation. The calculation model of the safe thickness of the antioutburst structure only calculates the thickness of the protection zone, but the value of the fracture zone depends on the accuracy of the geophysical results, leading to a large error in the results. In summary, there are still many typical and difficult problems in the study of the evolution mechanism of water inrush in deep tunnels.

Firstly, detonation pressure and excavation unloading are the important factors affecting the water inrush of deepburied rock mass. It is important to research the failure mechanism and seepage characteristics of rock mass under dynamic disturbance, strong unloading, and high osmotic pressure. It can reveal the mathematical relationship between stress, strain, seepage parameters, and groundwater flow variables. The establishment of a mathematical model of the evolution process of water inrush under the unloading disturbance and osmotic pressure is one of the difficulties in the study of the evolution mechanism of the inrush hydrodynamics.

Secondly, the deep burial depth and long tunnel line of tunnels bring high ground stress, strong osmotic pressure, and more complex regional seepage field evolution, making 
the catastrophe process of water inrush more complicated. Some research still needs improvement. Examples of these include the iterative method of seepage-stress-damage coupling of the calculation unit for transferring fluid pressure during rock mass rupture, the assignment method of transition conditions, and the boundary conditions between the fracture flow and the pipe flow model. There is no mature numerical method to simulate the water inrush.

Finally, the key control factors and corresponding control methods in the evolution process of water inrush and burst mud disasters under different disaster modes are still uncertain, making it difficult to establish corresponding decision-making models. Few scholars carry out research on the "control theory" of water inrush and burst mud disasters. It is an urgent need to carry out evolution mechanism of water inrush and disaster controlling method for collaborative research. A breakthrough is needed on the basic theoretical problem of the evolution mechanism with high pressure and large flow. It can effectively determine the key control factors and optimal control opportunities in different evolution stages of water inrush and provide theoretical support and method guidance for the active prevention and control of deep tunnel water inrush.

\section{Real-Time Monitoring Method of Catastrophe Precursor Information}

The evolution process of the water inrush disaster is a kind of dynamic damage process. The hydrothermal medium system, the hydrodynamic system, and the equilibrium state of surrounding rock mechanics changes sharply due to tunnel excavation, and the energy stored in the underground water is instantaneously released, carrying sediment and debris to the high speed of the excavation face [10]. It is a multifield coupling process integrating solid, liquid, and gas, which is accompanied by changes in information of multiple physical quantities. In view of the real-time monitoring of the evolution process of water inrush, the targets and contents of the water inrush disaster monitoring are clarified, and the fusion analysis is carried out under the coordination of multiple information monitoring to achieve effective disaster early warning.

3.1. Real-Time Monitoring Object for Water Inrush. During the construction of the tunnel, the static reserves of disaster sources can be detected through the geological forecast. The dynamic reserves of groundwater recharge can be estimated by the regional estimation of the supply channel [59], and the damage level can be quantified by the total quantity of water inrush. Through multi-information monitoring, the security situation of the antioutburst structure and the location of microseismic monitoring are analyzed. Finally, a comprehensive monitoring and early warning method based on the geological prediction of the disaster source, multiinformation fusion monitoring of the antioutburst structure, and microseismic location of the water inrush channel is established.

3.1.1. Anti-outburst Structure Monitoring. The antioutburst structure is the last barrier to the occurrence of water inrush disaster. In the process of water inrush evolution, a large amount of mechanical physics information will change on the antioutburst structure. A monitoring method for the fusion analysis of displacement (on the surface), force field (inside), and microseismic (in the far field) is proposed. Through the long-term stable monitoring of the water inrush channel and the evolution state, combined with the water inflow estimation, the time and space warning of water inrush are finally realized, as shown in Figure 1 [60].

Based on the multi-information characteristics of the seepage instability of water-blocking rock mass and the filling structure, the monitoring principle and design method of "microseismic-seepage dominant, multi-information fusion" are proposed. Combined with previous numerical simulation results, water inrush simulation test was carried out to obtain the fusion evolution law of precursor information of rock mass instability and water inrush. Aiming at the types of different typical water inrush disasters, the sensitivity ranking of multi-information is proposed, as shown in Table 2 , and the multi-information evolution model of different water inrush types can be obtained.

For the water inrush of the type of water-blocking rock mass destruction, the multi-information monitoring sensitivity of the microseismic information is stronger than other information because its antioutburst structure is fractured rock mass. During the process of rock mass rupture and stress redistribution, the microrupture develops inside the rock mass. The energy is released in the form of elastic waves, and the microseismic information is highlighted. In the water inrush caused by filling infiltration instability type, the antioutburst structure is mostly fault filling and pipeline filling. The brittle fracture of the medium is not obvious, resulting in the microseismic reaction that is not obvious, but the displacement information is sensitive during the evolution process.

3.1.2. Monitoring of Disaster Sources. The geological exploration technology is adopted to realize the spatial location and scale determination of the disaster source. The groundwater migration information is monitored, and the recharge water flow near the tunnel is estimated. The total amount of water inrush is the decisive factor in the classification of hazard levels, and it depends on the static and dynamic reserves of the disaster source. At present, the static reserves of disaster sources can be determined by advanced prediction methods such as induced polarization [61]. Determining the total amount of water inrush disasters and predicting the level of disaster damage can provide a theoretical basis for disaster control.

3.2. Monitoring of the Rupture Channel of the Anti-outburst Structure. As a kind of dynamic disaster, making full use of the information of a large number of mechanical and physical fields during the catastrophic process is the key to solving the problem of forecasting and warning of water inrush disasters. Therefore, a dynamic and static combined monitoring method is established by combining wireless sensing and cloud platform technology to realize 


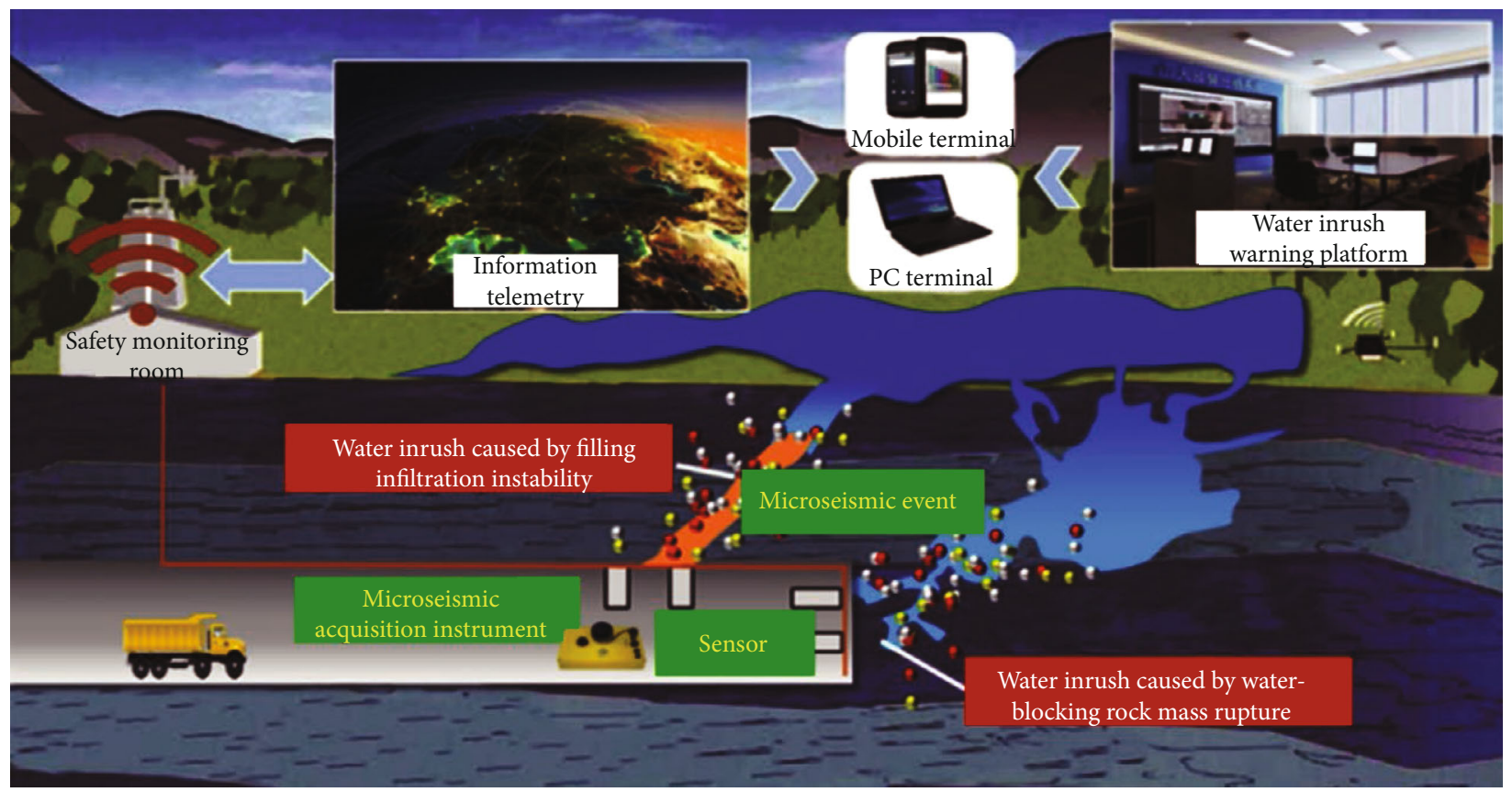

FIGURE 1: Microseismic monitoring design of antioutburst structure.

TABLE 2: Multi-information sensitivity ranking of water inrush [30].

\begin{tabular}{lccc}
\hline Type of disaster & Monitoring object & Information sensitivity ranking & Nature \\
\hline $\begin{array}{l}\text { Water inrush caused by } \\
\text { water-blocking rock mass } \\
\text { destruction }\end{array}$ & $\begin{array}{c}\text { Structure of fractured } \\
\text { rock mass }\end{array}$ & $\begin{array}{c}\text { Microseismic }>\text { stress field }>\text { displacement } \\
\text { field }>\text { seepage field }\end{array}$ & $\begin{array}{c}\text { Influence of seepage on } \\
\text { microseismic information } \\
\text { during rupture }\end{array}$ \\
$\begin{array}{l}\text { Water inrush caused by filling } \\
\text { infiltration instability }\end{array}$ & Fault filling, pipe filling & $\begin{array}{c}\text { Displacement field }>\text { osmotic pressure }>\text { stress } \\
\text { field }>\text { microseismic information }\end{array}$ & $\begin{array}{c}\text { Influence of disturbance } \\
\text { on the evolution process of } \\
\text { multi-information }\end{array}$ \\
\hline
\end{tabular}

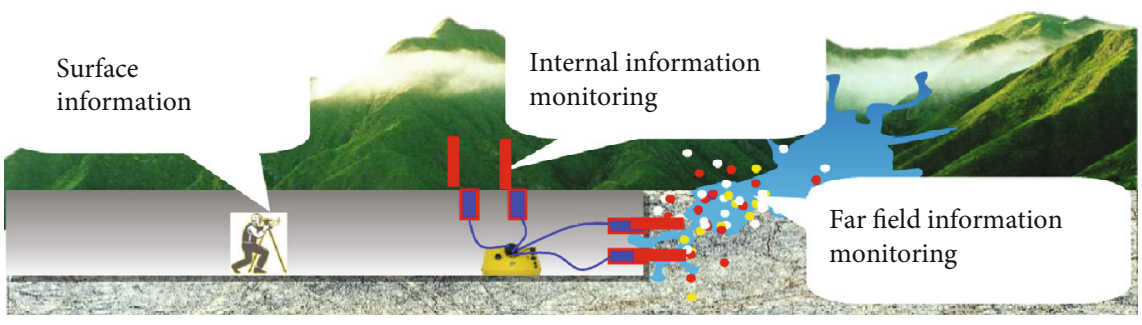

FIGURE 2: Dynamic monitoring of multifield information of water inrush.

surface information monitoring, internal information monitoring, and far field information monitoring in the disaster area, as shown in Figure 2. The surface information is mainly the displacement information of the surrounding rock surface, which can be obtained by monitoring and measuring means. The force field information such as stress and osmotic pressure inside the rock mass is monitored by sensor network in real time. At the same time, the remote field microseismic information monitoring in the disaster area is introduced, and a new multi-information monitoring method based on microseismic is proposed.
Through the research on multi-information monitoring and early warning methods of water inrush precursors in complex environment, the method of effective information identification and interference information elimination for water inrush precursors is proposed. Through the establishment of the basic theory of multi-information monitoring and early warning of the antioutburst structure and the comprehensive analysis of the evolution correlation and matching characteristics of each field of information to identify the evolution state of the disaster, the situation of the water inrush disaster is assessed. The time-oriented warning of the occurrence of the disaster is realized. 


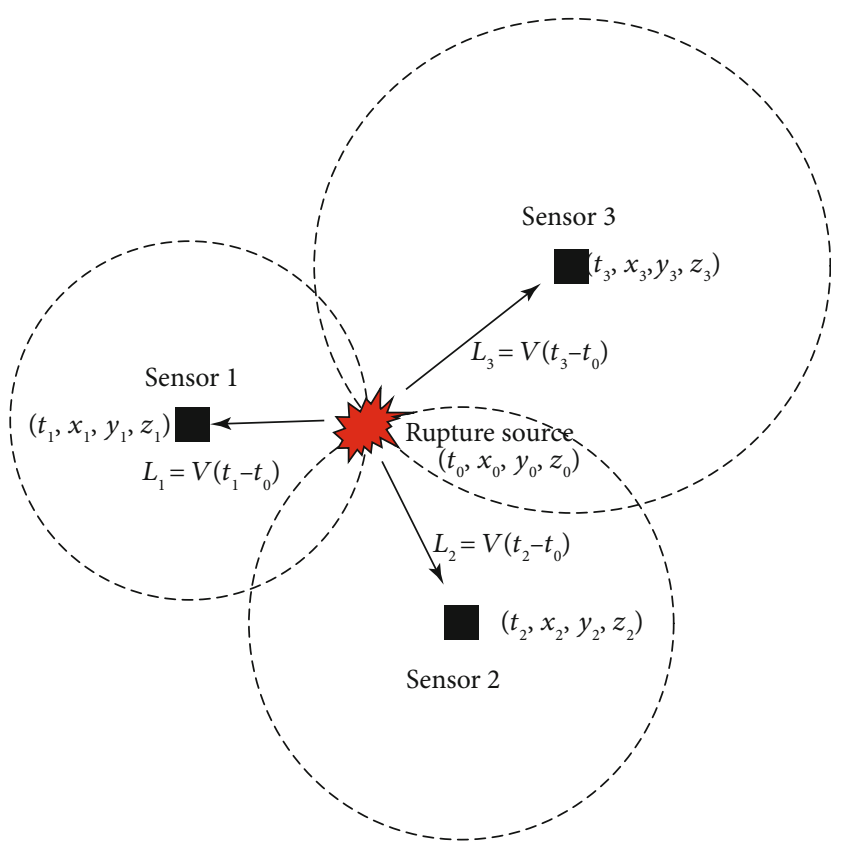

Figure 3: Microseismic event and positioning principle.

3.2.1. Positioning Method of Rupture Channel Based on Microseismic Monitoring. The research on monitoring and early warning of tunnel water inrush disaster shows that the water-blocking rock mass is anisotropic heterogeneous material. After the tunnel excavation disturbance, the stress redistribution occurs in the rock, so that the accumulated energy is released in the form of elastic waves. After the fluctuation is received by the sensor network, a shock response occurs, which is called a microseismic event. A large amount of microfracture leads to the penetration of cracks, which causes abnormal loosening of the original stable waterblocking surrounding rock. Therefore, the microseismic signal becomes an important precursor feature of dynamic disasters.

Figure 3 shows the positioning principle of the microseismic event. The sensor's point positions $x_{k}, y_{k}, z_{k}$ and the signal acquisition time $t_{k}$ by the sensor are known both in the initial construction and in the operation of the system. After measuring the original rock wave velocity $V_{P . S}$ by a blasting experiment, the path length of the microseismic signal collected by each sensor is shown as follows:

$$
\Delta L_{k}=V_{P \cdot S} \times \Delta t_{k}=V_{P \cdot S} \times\left(t_{k+1}-\Delta t_{k}\right) .
$$

The distance from each sensor to the source point can be expressed as follows:

$$
\Delta L_{k}=\sqrt{\left(x_{k}-x\right)^{2}+\left(y_{k}-y\right)^{2}+\left(z_{k}-z\right)^{2}} .
$$

By arranging the sensor array in the tunnel, the automatic acquisition, transmission, and processing of the microseismic data can be realized. The location of the internal damage of the rock is determined by the microseismic positioning principle and is displayed on the three-dimensional map.

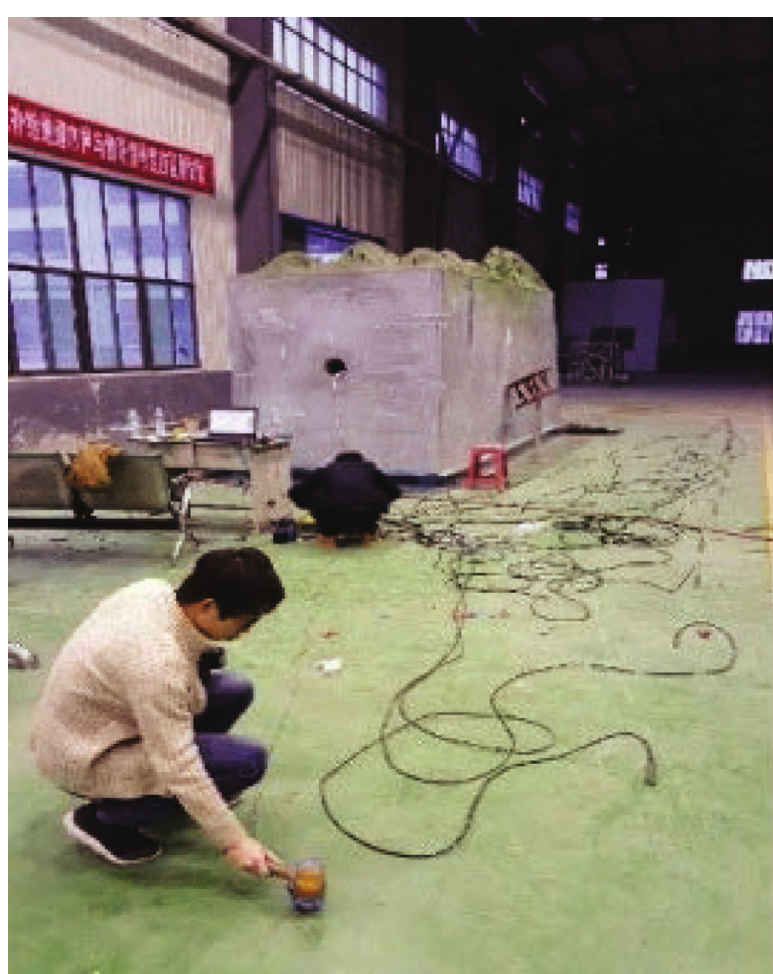

Figure 4: Model test of microseismic positioning.

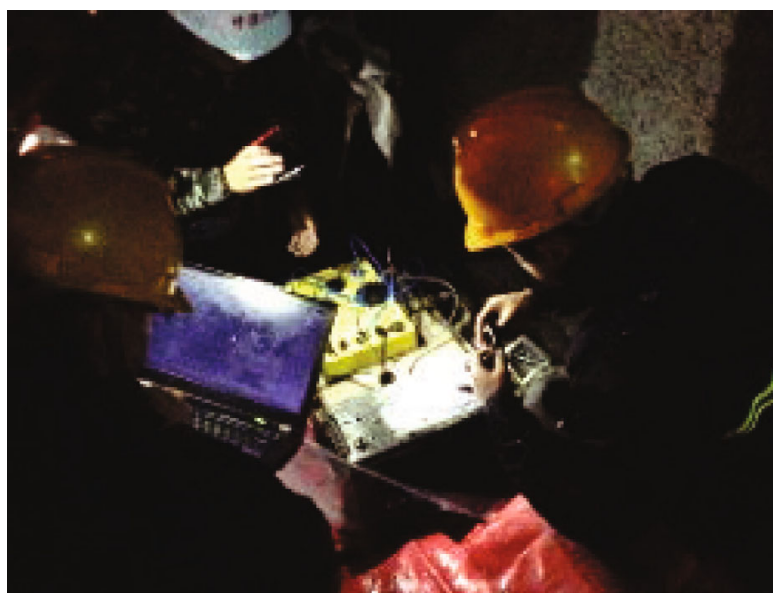

FIgURE 5: Field verification of upgrades for microseismic equipment.

The microseismic monitoring technology has the characteristics of long-distance overall monitoring and $3 \mathrm{D}$ real-time monitoring, which achieves the purpose of monitoring the stability of the water-blocking rock mass.

\subsubsection{Improved Microseismic Technology for Monitoring} Water Inrush Disasters. The traditional microseismic monitoring technology is mostly used for monitoring and early warning of rockburst disasters in underground engineering. There are few applications for monitoring antioutburst structure in the water inrush disaster. As shown in Figure 4, the model test method is used to compare and 


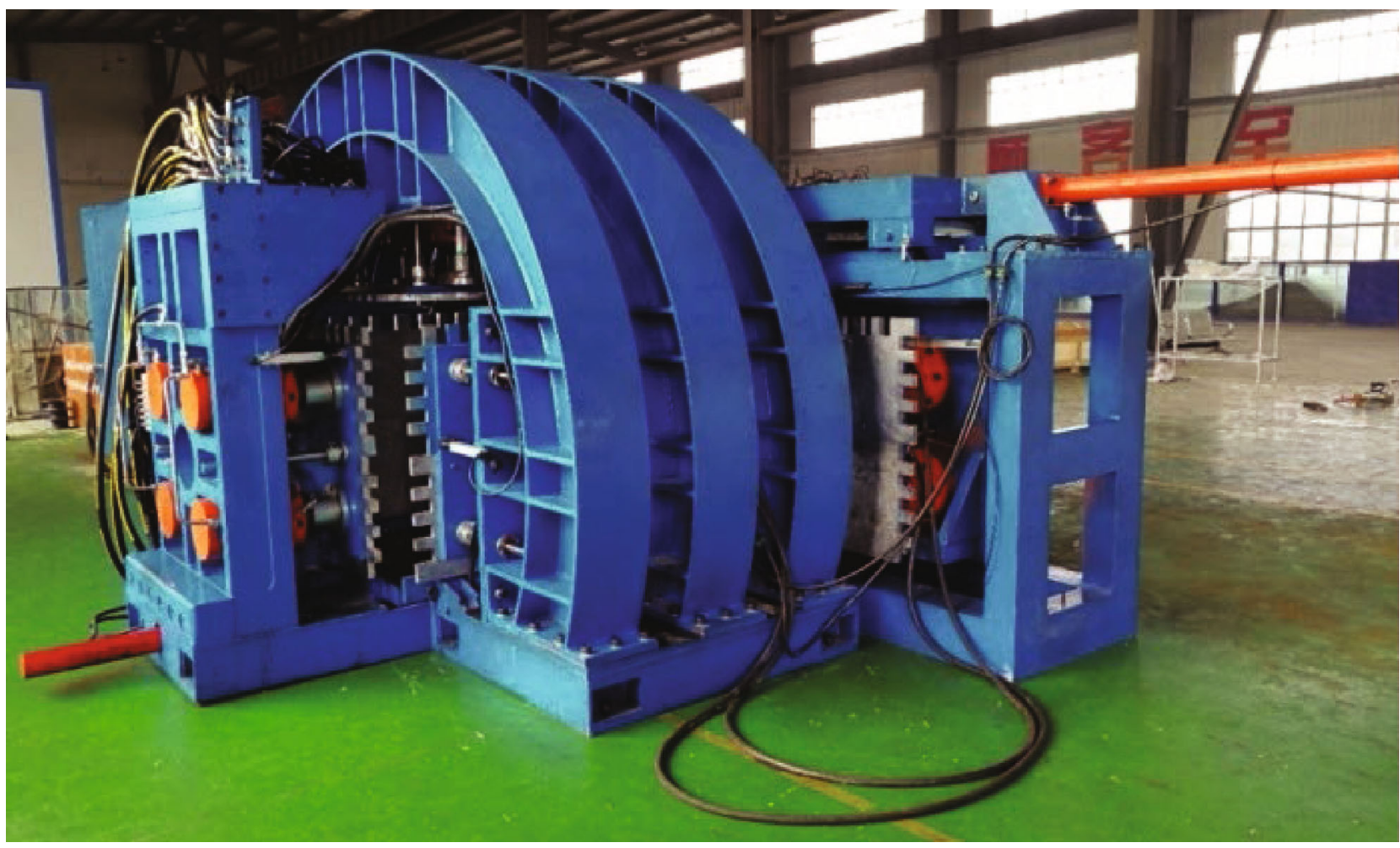

FIGURE 6: Model test and precursor information collection of water-blocking rock mass [30].

analyze the microseismic response law during the process of rupture of dry rock mass and water-rich rock mass, and provide a basis for exploring the difference between rock burst disaster and water inrush disaster [62]. Through the field application of microseismic technology in the water inrush disaster of tunnel engineering, the difference of the monitoring signal between the water inrush disaster and the rock burst disaster is found, as shown in Figure 5. The traditional microseismic monitoring system could not be directly applied to the monitoring of the water inrush disaster.

The parameters of the monitoring hardware are improved by upgrading the microseismic monitoring system from the software layer and the hardware layer, which is different from the conventional rockburst monitoring. The software is embedded in the filter program for rock cracking signal of water-rich mass. At the same time, in the aspect of data processing, the self-developed multi-information fusion analysis software TMIWS is embedded in the microseismic information processing system. It realizes the dual function of the identification of precursor information and the location of the water inrush channel.

\section{Multi-Information Fusion Analysis and Early Warning Technology}

\subsection{Matching Model of Multitype Water Inrush}

4.1.1. Multifield Information Evolution Law of Rupture in Water-Bearing Rock Mass. The physical basis of monitoring and early warning of water inrush information is a large amount of precursor information generated during the

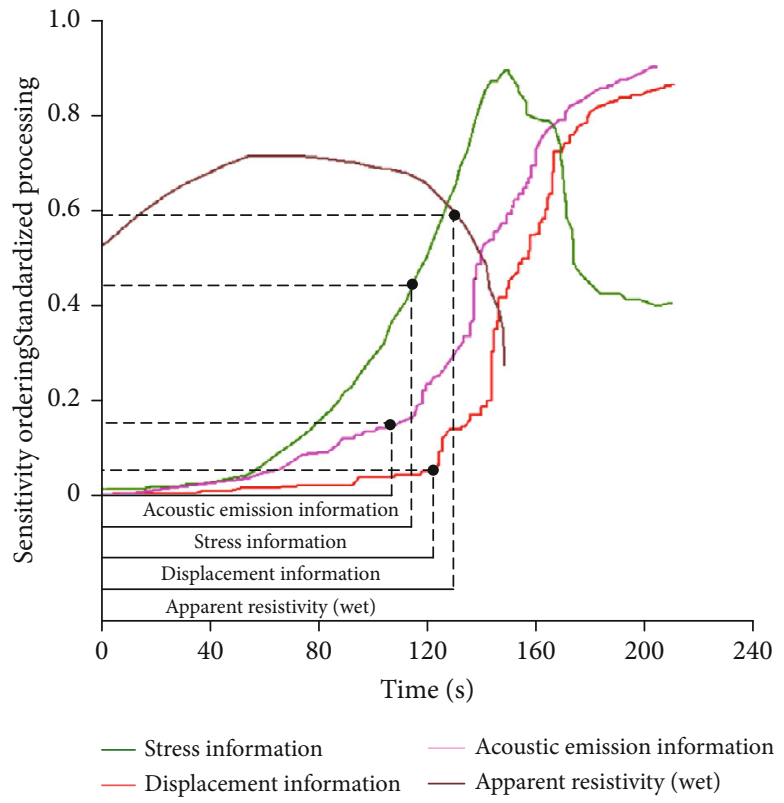

FIGURE 7: Rock mass fracture multiphysical parameter sensitivity ranking [30].

instability of rock mass. These precursor information includes acoustic emission, apparent resistivity, stress, and displacement, as shown in Figure 6. It is more difficult to obtain all of this information accurately during the actual tunnel construction process, but the occurrence of this information is actually present. 


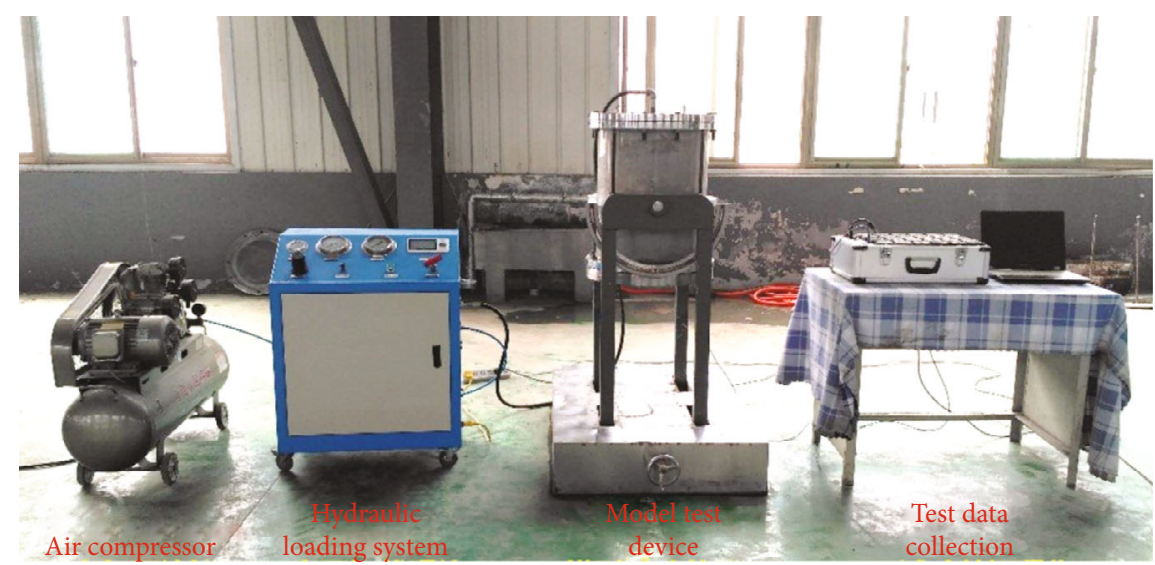

FIgURe 8: Physical simulation test system for seepage instability of water in the filling medium [30].

Through the model test of the rupture state of the tunnel surrounding rock, and combined with the numerical simulation results of the predecessors, the evolution law of the precursory information of the rock mass instability and water inrush is obtained and the multiphysical parameter sensitivity ranking of the rock mass rupture is performed, as shown in Figure 7. The sensitivity ranking of the critical points of the precursory information of the water inrush rock damage is acoustic emission information $>$ stress information > displacement information $>$ osmotic pressure information.

4.1.2. Evolution Law of Multifield Information of Water Inrush of Infiltration Instability. During the tunnel construction process, with the influence of the water-induced disaster-causing structure, as the tunnel surface is gradually approaching the water-induced disaster-causing structure, the water inrush channel gradually penetrates. When the excavation disturbance continues to occur, a large amount of water infiltrates into the tunnel. When the exposed structure is a filling medium, the filling medium is unstable and water inrush occurs. Figure 8 is a physical simulation test system for seepage instability and water inrush in tunnel filling medium.

The filling medium has less stress redistribution effect than the rock mass. Under the action of excavation disturbance, the filling structure is easy to deform. The internal stress redistribution is mainly concentrated in the weak boundary position, and the large-scale stress change occurs at the boundary, causing the slippage of the entire filling body or deformation near the dominant seepage channel. The sensitivity rankings of the precursor information are seepage information, displacement information, stress information, and acoustic emission information, as shown in Figure 9. In the construction process of the tunnel, combined with the results of the simulation test, the time node of the water quality changing from turbid to clear can be used as a discontinuity of precursor information for the seepage instability.

4.2. Time-Oriented Warning Based on Multi-Information Fusion Analysis. The "five factors" that affect the occurrence

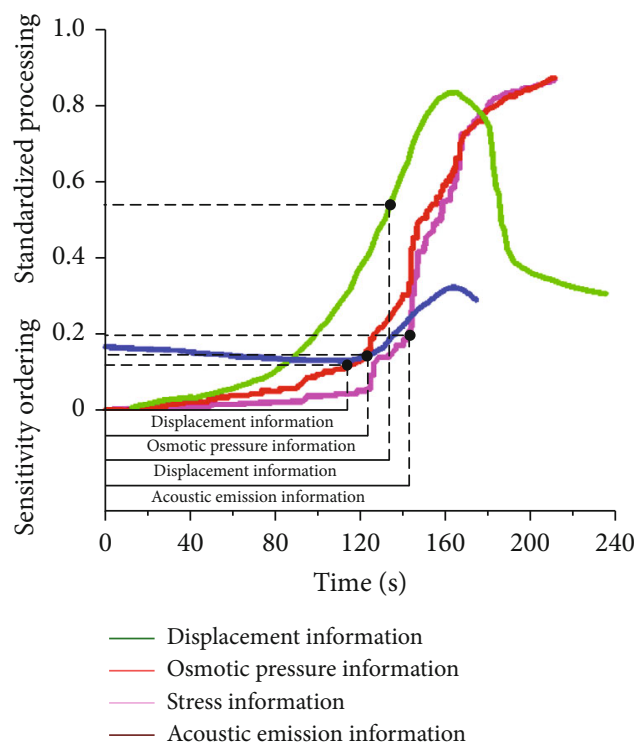

FIgURE 9: Classification of filling osmotic instability multiphysical parameters sensitivity [30].

of water inrush are physical properties, structure, stress, energy, and time. Based on these factors and the real-time monitoring data such as microseismic, displacement, stress, osmotic pressure, temperature, and resistivity, the situation assessment theory $[63,64]$ is introduced into the disaster prediction of water inrush. Using the method of log auditing $[65,66]$, real-time monitoring and correction of multiheterogeneous information is used to establish the water inrush potential prediction model. In view of the disadvantages of the Yager formula, based on the previous D-S fusion theory, an average weighted fusion algorithm of the probability of conflict of evidence is proposed. The distribution function of the probability of conflict of evidence is weighted according to the average support degree of each proposition and then distributed. Finally, a new D-S fusion theory is proposed. This method overcomes the shortcomings of the previous methods and forms a multivariate information fusion analysis method for the precursor of water surge. Based on the improved D-S fusion theory, a situational 


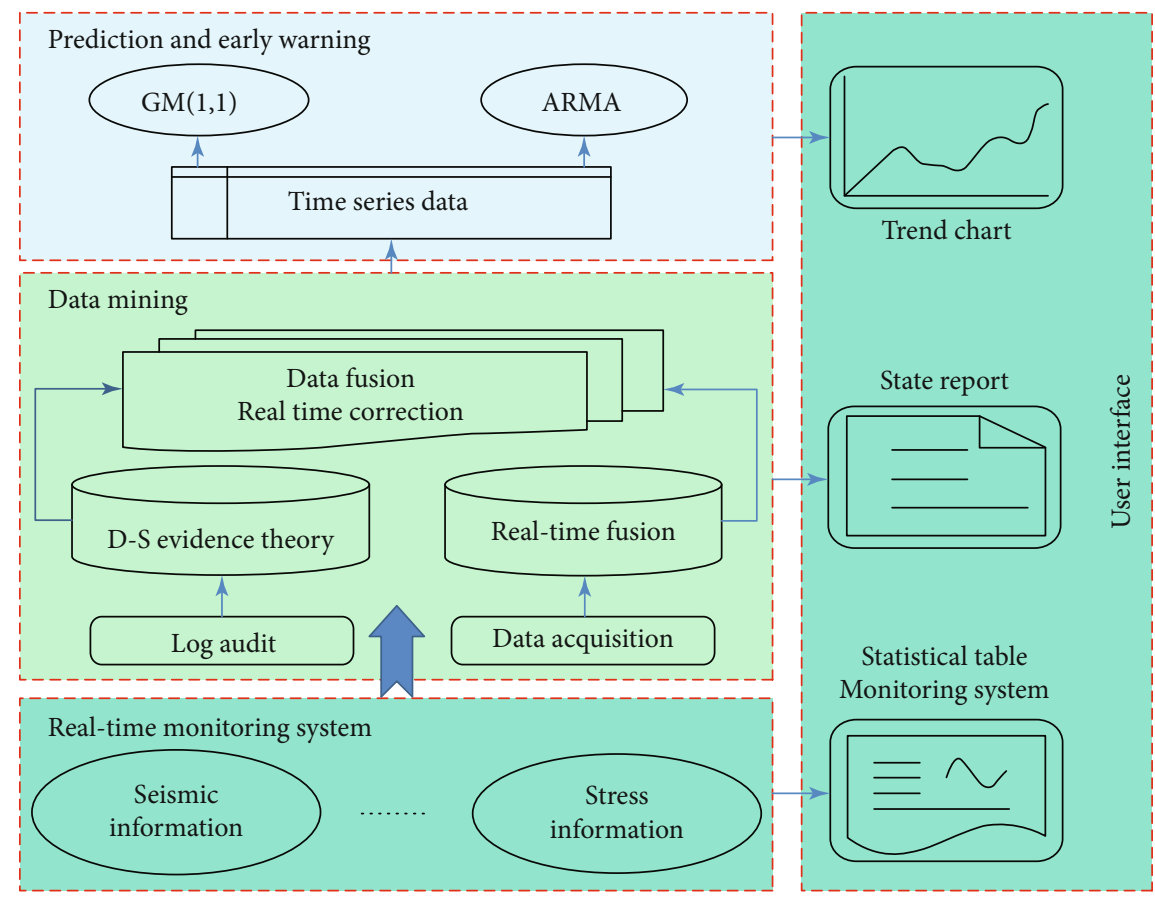

FIGURE 10: Fusion prediction method of water inrush situation.

fusion prediction method for water inrush predicting is proposed. Using this fusion prediction method, the water inrush condition is obtained in continuous time. The time tendency of the occurrence of a water inrush disaster is finally determined.

The implementation process of fusion prediction method of water inrush situation is as follows. First, the monitoring data and construction log obtained in the monitoring system such as microseismic, displacement, stress, osmotic pressure, temperature, and resistivity are converted into time series information. Secondly, during the entire information change process, a sliding window of $\Delta T$ (time) is set (the trailing edge of the sliding window is the real-time display time, the size of $\Delta T$ is freely set according to actual needs). Using the $\log$ audit method, the probability of occurrence of water inrush in the $\Delta T$ is quantified. Finally, multiphysical data is fusion analyzed by introducing the D-S fusion theory. The real-time data of the monitoring system corrects the fusion analysis results to meet the prediction of the change of the water inrush state. Based on the accurate prediction, the real-time status is obtained which fully reflects the actual situation of water inrush. Because the result of the water inrush prediction is a time series, the linear visual display is realized by using the $\operatorname{GM}(1,1)$, ARMA, and Holt-Winters model to predict the probability of the water inrush, as shown in Figure 10.

All the water inrush expectations are fused analysis by using improved D-S evidence fusion methods [30] to obtain the occurrence probability of water inrush. The precursory multi-information accompanying the occurrence of tunnel water inrush disaster includes microseismic, displacement, stress, osmotic pressure, temperature, and apparent resistivity. The information data that can be obtained in the actual construction process is limited. The formula for synthesizing the probability of occurrence of water inrush in three types of physical quantity monitoring systems is deduced.

$$
\left\{\begin{array}{l}
m(\phi)=0, \\
m(A)=\prod_{i d=1}^{5} m_{i d}(A)+\lambda \cdot f(A), \\
m(\bar{A})=1-m(A), \\
m(H)=0,
\end{array}\right.
$$

where

$$
\begin{aligned}
\lambda & =1-\prod_{i d=1}^{5} m_{i d}(A)-\prod_{i d=1}^{5} m_{i d}(\bar{A}), \\
f(A) & =\frac{1}{5} \sum_{i d=1}^{5} m_{i d}(A) .
\end{aligned}
$$

According to equation (6), the alarm log information data of the multiphysical monitoring system is analyzed by fusion to obtain the occurrence probability of water inrush, $\delta_{h p}=m(A)$.

Through the data mining of the precursor multiinformation of water inrush in the $\Delta T$, the real-time monitoring data of the around $\Delta T$ time are corrected to obtain the time series-based water inrush situation. Therefore, the water inrush situation can be predicted by $\operatorname{GM}(1,1)$. The $\operatorname{GM}(1,1)$ is the content of the grey system theory. It can find the regular and make predictions in a limited number of 


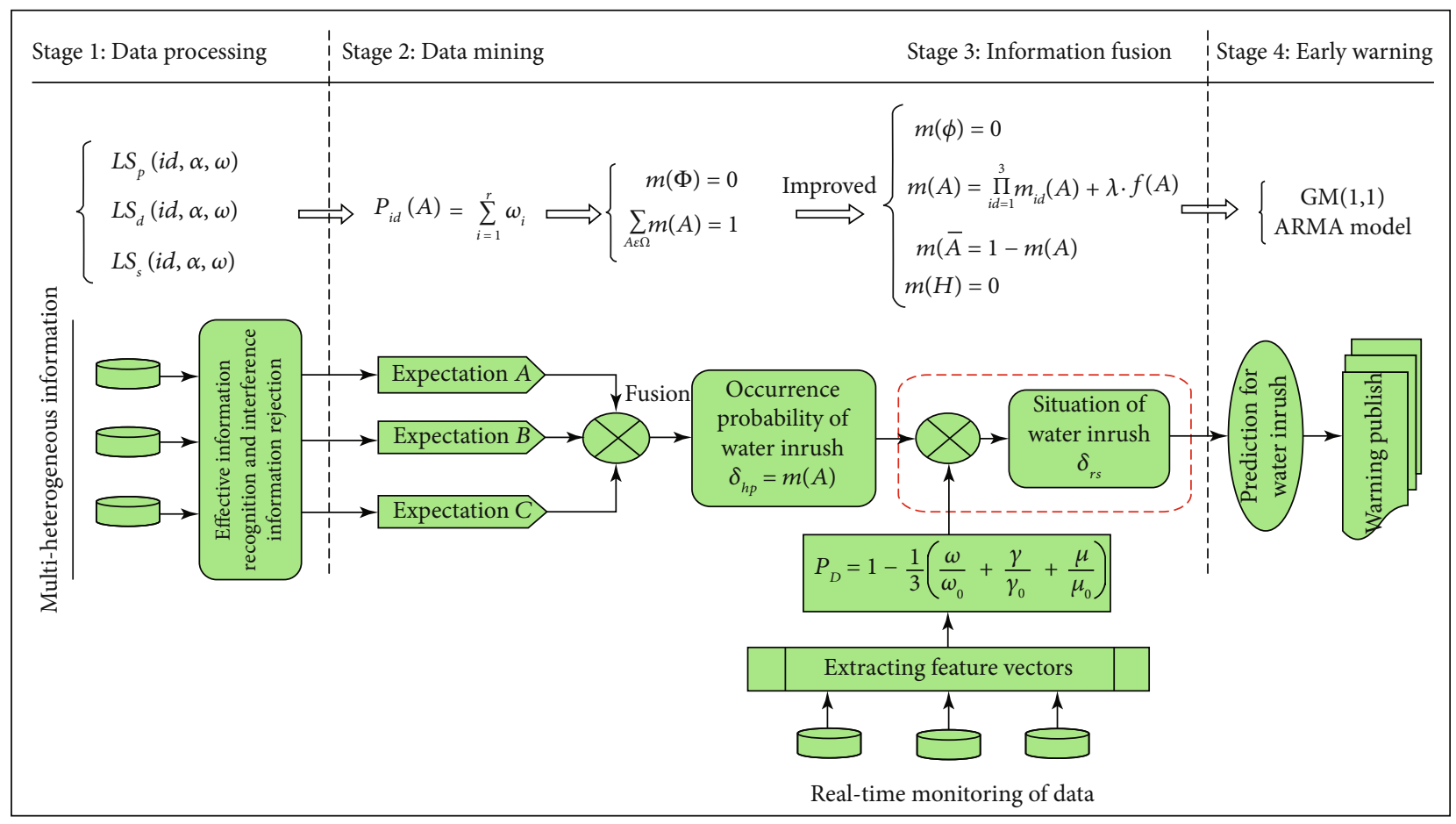

FigURE 11: Fusion prediction model of water inrush.

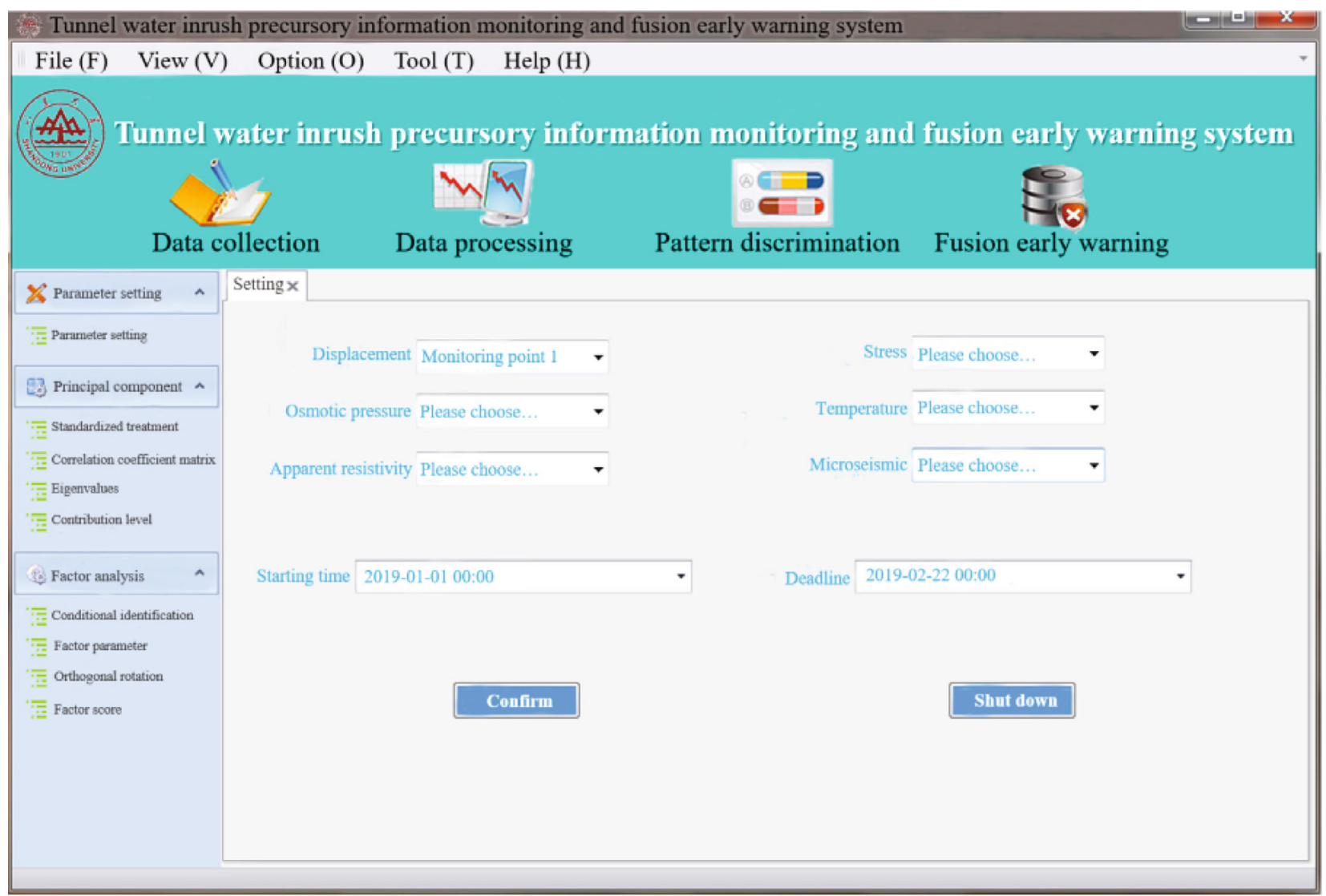

FIGURE 12: Monitoring and integration warning system for precursor information of water inrush [30]. 

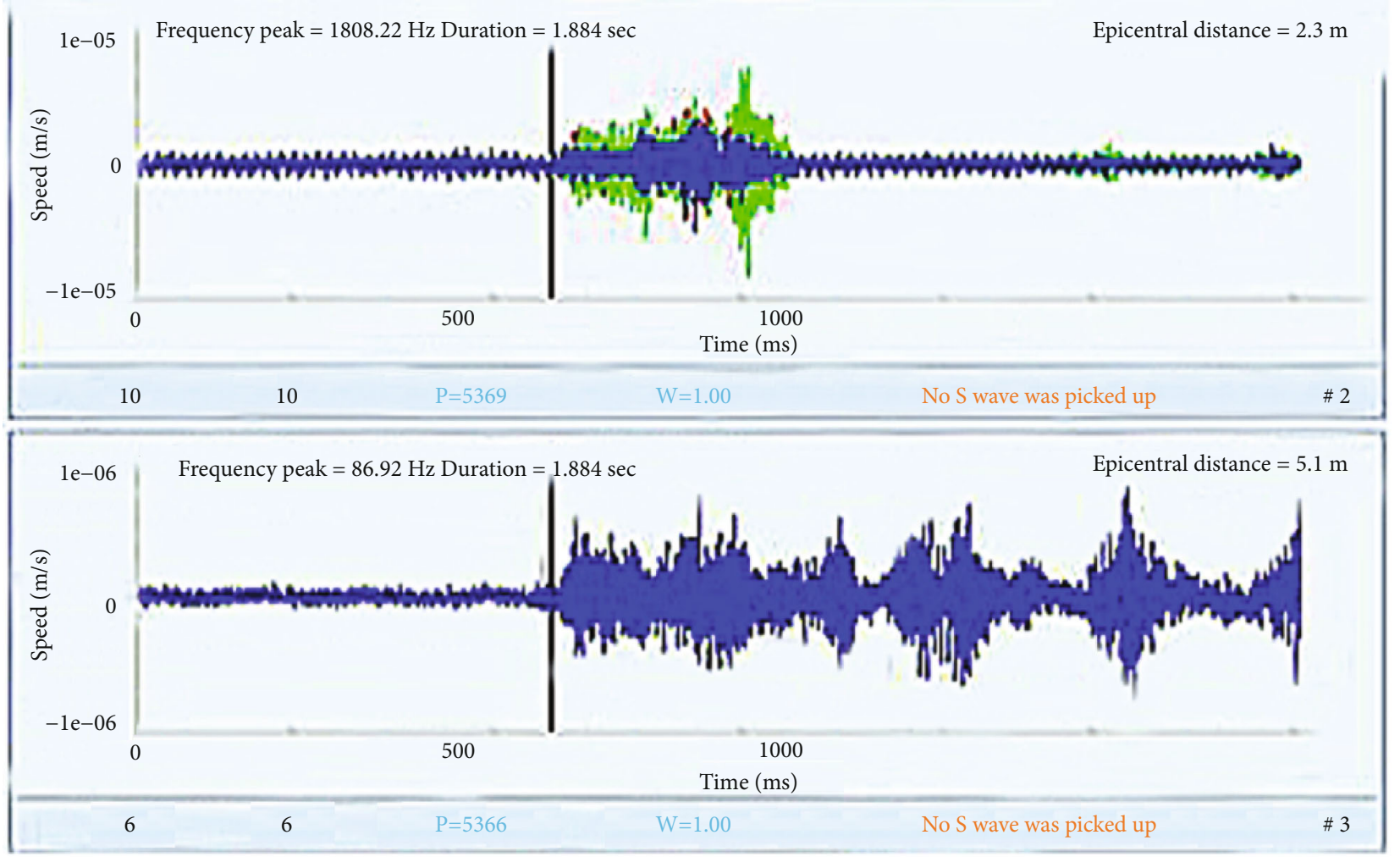

Figure 13: The microseismic monitoring signal of water inrush.

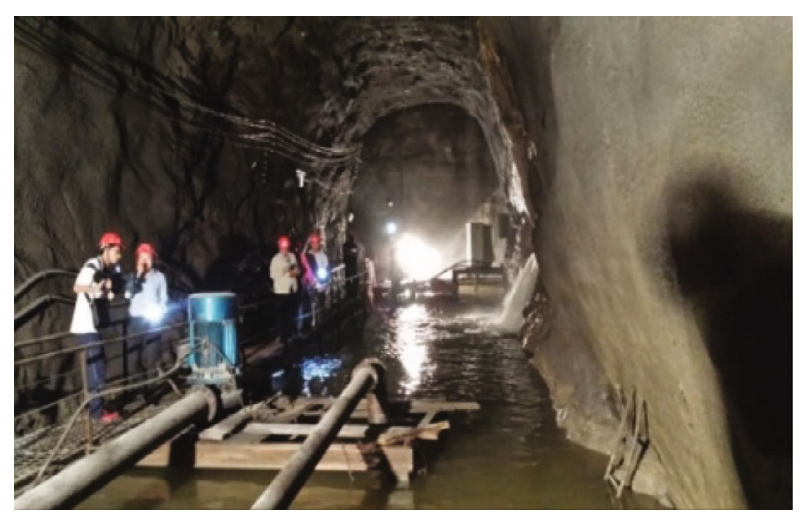

FIGURE 14: On-site water discharge.

data or discrete data, and draw the situational curve for visual expression. It also can assist the engineer to fully understand and grasp the development trend of the tunnel water inrush situation. A propensity warning for the occurrence of water inrush is achieved, as shown in Figure 11.

4.3. The Early Warning of Water Inrush Based on Microseismic Monitoring. If there are many recharge channels near the disaster source of the water inrush, when a water inrush disaster occurs, the water inrush channel will have a large amount of water supply. The turbulence signal generated by the water flow impinging on the channel wall can propagate in the anisotropic rock soil medium. Through

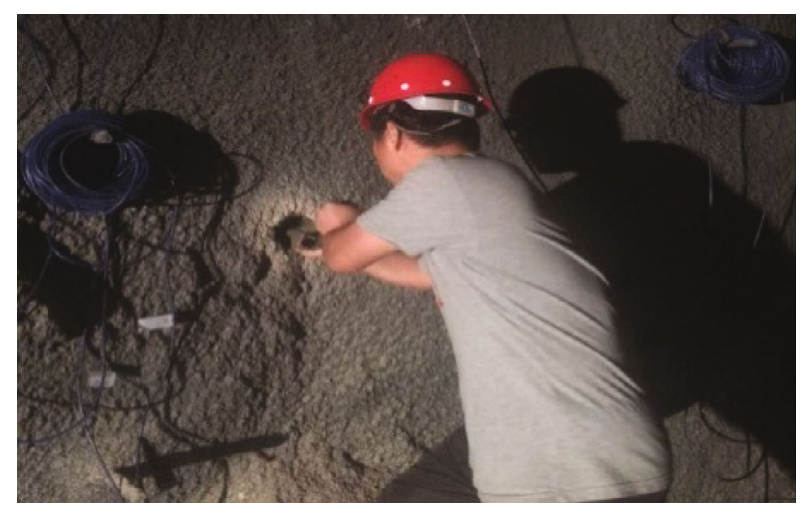

FIGURE 15: Monitoring component installation.

the simulation test, the feasibility of capturing signals and inverting the positioning supply channel in the tunnel surface was verified. Therefore, a hydroacoustic detector and a microseismometer suitable for the monitoring of water inrush disasters of tunnel have been developed.

The microseismic monitoring equipment is combined with the wireless sensor network to form a real-time monitoring method for the water inrush multi-information carried by microseismic. Through the quantitative analysis of the multi-information situation, based on the established multi-information depth fusion algorithm, the situation of water inrush and the location of rupture channel are predicted to realize the continuous real-time monitoring and 


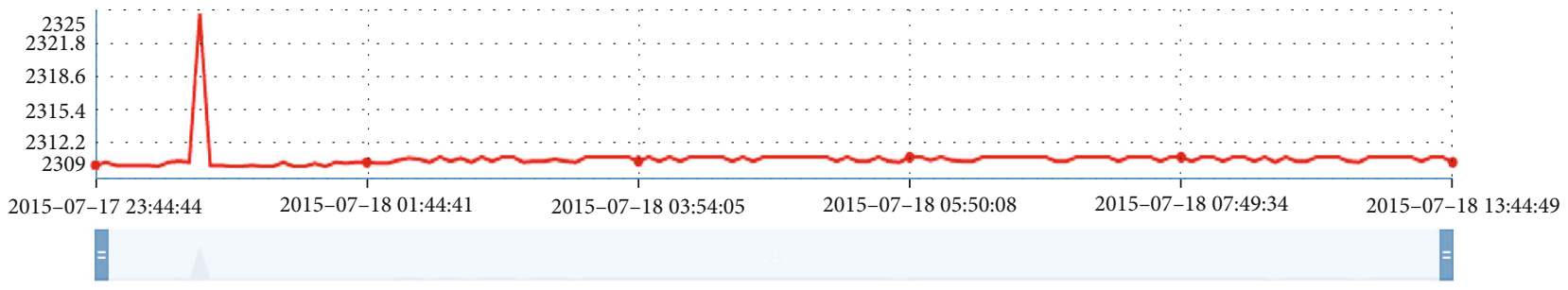

(a) Pore water pressure

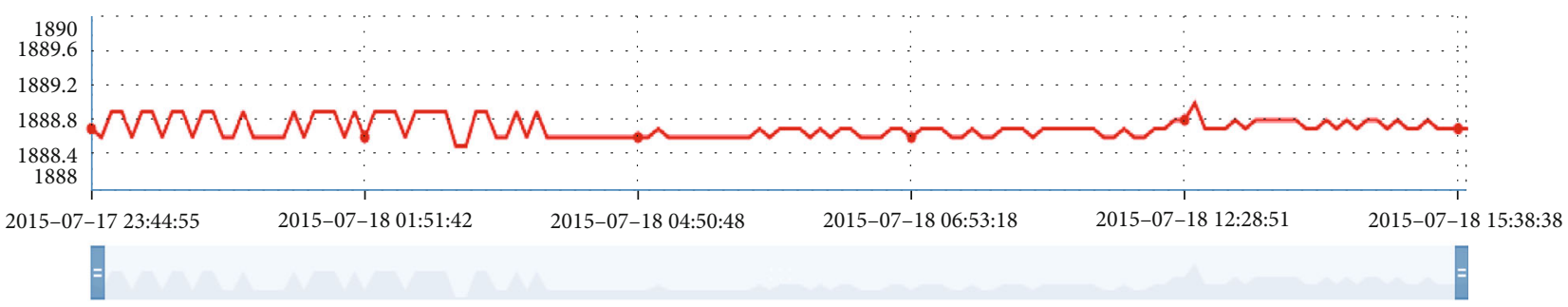

(b) The stress curve

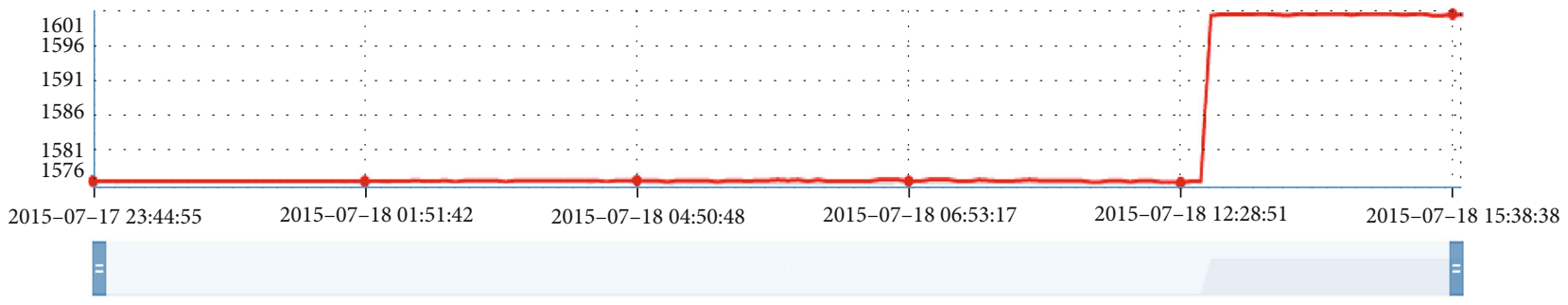

(c) The displacement curve

Figure 16: Monitoring data of physical quantity.

early warning of water inrush disaster, as shown in Figures 12 and 13.

During the tunnel construction process, disaster source static reserves are detected through the whole process of geological prediction. The dynamic reserve of groundwater recharge is estimated by the regional estimate of the recharge channel. The hazard level is quantified by the amount of water in the water inrush. Through the multi-information monitoring of the antioutburst structure, the analysis of the security situation of the antioutburst structure to determine the time tendency, and the microseismic monitoring to determine the location of the water inrush channel, a comprehensive monitoring and early warning method was established, based on the geological prediction of the disaster source, the multi-information fusion monitoring of the antioutburst structure, and the microseismic positioning of water channels.

\section{Engineering Applications}

The Yuelongmen tunnel of Sichuan Chenglan Railway is one of the most difficult railway projects in China. It is located in the alpine valley area on the eastern edge of the QinghaiTibet Plateau. Mountains and steep valleys surround it. It passes through Jiuzhaigou and the Giant Panda Protection Base. Accompanied by the collision between the Indian plate and the Eurasian plate, the topographical and geological conditions show typical "four poles and three highs" charac- teristics. The Yuelongmen tunnel is a double-hole tunnel with a total length of $19981 \mathrm{~m}$ for the left line and $20042 \mathrm{~m}$ for the right line. The tunnel passes through the Longmenshan active fault zone and fault fracture zone. The maximum depth of the tunnel is $1445 \mathrm{~m}$. In the process of construction, disasters such as large deformation and water inrush frequently occur under high ground stress. In the D2K94 $+645 \sim \mathrm{D} 2 \mathrm{~K} 94+675$ mileage, through the deployment of monitoring sensors, video monitoring devices, and remote wireless transmission systems, a multi-information monitoring platform based on microseismic is built to monitor realtime multivariate precursor information, as shown in Figures 14 and 15. During the construction period, 27 small and medium-sized water inrush were accurately warned, and the water inrush situation was effectively controlled. The estimation method of the hydrostatic volume and dynamic reserves of the disaster source was proposed, and the hazard level warning was realized, ensuring safe construction in water. It guarantees the safe construction of the extremely high-risk section of the Yuelongmen tunnel and promotes its application throughout the entire railway.

The remote monitoring and early warning analysis system for major water inrush includes data acquisition, data processing, pattern discrimination, and fusion warning. Based on the remote real-time acquisition of physical quantity information to find the evolution law of precursor information of two kinds of water inrush, the water inrush pattern recognition and discrimination model was 
established to carry out accurate type identification of water inrush, as shown in Figure 16. A fusion analysis model of tunnel water inrush was established. Through the analysis of multi-information fusion, the evolution state of the water inrush was predicted in time. In space, the early warning technology of microseismic positioning channel and integrated multi-information prediction situation was used as an auxiliary to monitor and warn. Multiterminals, such as total monitoring center, on-site subcenter, base station, and internet, have realized real-time monitoring and early warning of tunnel water inrush in different places.

\section{Conclusions}

This paper systematically summarizes the failure mode, the occurrence criterion, and the safe thickness determination method of the antioutburst structure and expounds the catastrophic evolution process of the formation of the water inrush channel. The disaster source and antioutburst structure of water inrush were monitored in real-time to obtain the evolution law of precursory multi-information. Finally, the identification method of the precursor information of water inrush was established, and the propensity prediction of water inrush time was realized. The remote monitoring and early warning analysis system of major water inrush was established, which can provide the basis for prevention and control of disasters.

(1) The catastrophic evolution mechanism of the progressive failure of the resistance structure and the infiltration and instability of filling medium was explained. By systematically summarizing the existing theoretical model of water inrush, calculation method, and applicable conditions, this paper focuses on the hot and difficult problems, key breakthrough directions, and development trends of the mechanism research of water inrush. The research on the dynamic evolution mechanism of water inrush in deep tunnels provides theoretical support and method guidance for the active prevention and control of water inrush

(2) By comparing the application of microseismic monitoring in rockburst and water inrush, the improvement schemes of software and hardware for traditional microseismic technology of antioutburst structure were proposed to locate the rupture of the antioutburst structure. The scheme was embedded in the fusion early warning software. Then, the cluster analysis theory was used to realize the effective acquisition of precursory information of water inrush. The identification problem of the evolution of state and precursor information of water inrush was solved

(3) An improved D-S fusion method was introduced for the fusion analysis of multidimensional heterogeneous information. Combined with real-time monitoring data to correct the fusion analysis results, the tendency to predict the water inrush time was real- ized. Besides, the true state of the water inrush situation was comprehensively reflected. Considering the estimation of the static reserve and the dynamic reserves under the water supply replenishment of disaster sources, it can achieve the magnitude prediction of the water inrush. Finally, a monitoring and early warning analysis system for major water inrush was established

(4) Yuelongmen tunnel relies on the multi-information collection wireless transmission platform to establish a multilevel and comprehensive early warning system. It realized data cloud storage analysis and real-time monitoring and early warning of multiterminals. It realizes the time and space warning of major water inrush and promotes the development of active prevention and control of disasters

\section{Data Availability}

The data used to support the findings of this study are included within the article.

\section{Conflicts of Interest}

The authors declare that they have no conflicts of interest.

\section{Acknowledgments}

The research for this paper was supported by a project of the National Science Foundation for Distinguished Young Scholars of China (Grant No. 52025091), the National Natural Science Foundation of China (Grant Nos. 51909143, 52009076, and 52009070), a project of the Joint Funds of the National Natural Science Foundation of China (Grant No. U1934218), the Shandong Provincial Key Research and Development Program (Major Scientific and Technological Innovation Project) (Grant Nos. 2019JZZY010601 and 2019JZZY010428), and the China Postdoctoral Science Foundation (Grant Nos. 2020M682182 and 2021T140414).

\section{References}

[1] R. Q. Huang and X. N. Wang, "Analysis of major disaster geological problems in deep tunnel engineering," Hydrogeological Engineering Geology, vol. 4, pp. 21-24, 1998.

[2] X. W. Luo and F. L. He, "A study of geological structures inclined to disaster and models of water burst in deep-buried long tunnels," Modern Tunnelling Technology, vol. 51, no. 1, pp. 21-25, 2014.

[3] M. S. Wang and Z. S. Tan, "China tunnel and underground construction technology," Strategic Study of CAE, vol. 12, no. 12, pp. 4-10, 2010.

[4] D. Y. Chen, Study on water inrush mechanism and real-time monitoring method of karst cave in tunnels [Ph.D. thesis], Shandong University, Jinan, 2016.

[5] Y. G. Wang, Study on scourge and prevention of karst tunnel water inrush [Ph.D. thesis], China Academy of Railway Sciences, Beijing, 2010. 
[6] Z. W. Liu, Karst waterburst mechanism and prevention countermeasures in Yuanliangshan tunnel [Ph.D. thesis], China University of Geosciences, Beijing, 2004.

[7] Z. W. Liu, M. C. He, and S. R. Wang, "Study on karst waterburst mechanism and prevention countermeasures in Yuanliangshan tunnel," Rock and Soil Mechanics, vol. 27, no. 2, pp. 228-232, 2006.

[8] J. X. Wang, B. Feng, and X. S. Zhang, "Hydraulic failure mechanism of karst tunnel surrounding rock," Chinese Journal of Rock Mechanics and Engineering, vol. 29, no. 7, pp. 13631370, 2010.

[9] J. Q. Guo and C. S. Qiao, "Study on water inrush mechanism and thickness of rock wall of karst tunnel face," Journal of The China Railway Society, vol. 34, no. 3, pp. 105-111, 2012.

[10] L. P. Li, Study on evolution mechanism and application of water inrush disaster in high risk karst tunnel [Ph.D. thesis], Shandong University, Jinan, 2009.

[11] L. P. Li, D. Y. Chen, and S. C. Li, "Numerical analysis and fluid-solid coupling model test of filling-type fracture water inrush and mud gush," Geomechanics and Engineering, vol. 13, no. 6, pp. 1011-1025, 2017.

[12] Z. L. Nie, Analysis for water burst mechanism and study on mechanical features of tunnel lining in water filled karst area [Ph.D. thesis], Southwest Jiaotong University, Chengdu, 2009.

[13] Z. Q. Zhou, L. P. Li, S. S. Shi et al., "Study on tunnel water inrush mechanism and simulation of seepage failure process," Rock and Soil Mechanics, vol. 41, no. 11, pp. 3621-3631, 2020.

[14] R. Q. Huang and X. N. Wang, "Analysis of hydraulic splitting in deep water tunnel gushing process," Chinese Journal of Rock Mechanics and Engineering, vol. 19, no. 5, pp. 573-573, 2000.

[15] X. J. Weng, Study on inrush of water and mud mechanism and grouting control technology in water-rich fault fracture zone of tunnel [Ph.D. thesis], Shandong University, Jinan, 2014.

[16] J. W. Zhang, Y. Tan, and T. Chen, "Study on mechanism of batholite water inrush in lateral karst tunnel," Journal of Highway and Transportation Research and Development, vol. 34, no. 5, pp. 109-115, 2017.

[17] S. Q. Sun, P. He, G. Wang et al., "Shape characterization methods of irregular cavity using Fourier analysis in tunnel," Mathematics and Computers in Simulation, vol. 187, pp. 191214, 2021.

[18] X. Zhang, D. J. Sanderson, and A. J. Barker, "Numerical study of fluid flow of deforming fractured rocks using dual permeability model," Geophysical Journal International, vol. 151, no. 2, pp. 452-468, 2002.

[19] L. Jing, Y. Ma, and Z. Fang, "Modeling of fluid flow and solid deformation for fractured rocks with discontinuous deformation analysis (DDA) method," International Journal of Rock Mechanics and Mining Sciences, vol. 38, no. 3, pp. 343-355, 2001.

[20] L. Q. Ma, D. S. Zhang, and X. X. Miao, "FLAC3D simulation of seepage law of mining rock mass," Journal of Hunan University of Science and Technology (Natural Science Edition), vol. 21, no. 3, pp. 1-5, 2006.

[21] L. C. Li, C. A. Tang, and Z. Z. Liang, "Numerical analysis of pathway of groundwater inrush from faults in coal seam floor," Chinese Journal of Rock Mechanics and Engineering, vol. 28, no. 2, pp. 290-297, 2009.

[22] C. A. Tang, T. H. Yang, L. C. Li et al., "Numerical simulation of influence of pore water pressure on rock crack propagation," Rock and Soil Mechanics, vol. 24, no. S2, pp. 17-20, 2003.
[23] Z. Q. Zhou, P. G. Ranjith, W. M. Yang, S. S. Shi, C. C. Wei, and Z. H. Li, "A new set of scaling relationships for DEM-CFD simulations of fluid-solid coupling problems in saturated and cohesiveless granular soils," Computational Particle Mechanics, vol. 6, no. 4, pp. 657-669, 2019.

[24] Z. Q. Zhou, Z. H. Li, C. L. Gao et al., "Peridynamic microelastoplastic constitutive model and its application in the failure analysis of rock masses," Computers and Geotechnics, vol. 132, article 104037, 2021.

[25] C. L. Gao, Z. Q. Zhou, Z. H. Li, L. P. Li, and S. Cheng, "Peridynamics simulation of surrounding rock damage characteristics during tunnel excavation," Tunnelling and Underground Space Technology, vol. 97, article 103289, 2020.

[26] S. C. Li, C. L. Gao, Z. Q. Zhou et al., "Analysis on the precursor information of water inrush in karst tunnels: a true triaxial model test study," Rock Mechanics and Rock Engineering, vol. 52, no. 2, pp. 373-384, 2019.

[27] Y. C. Mo, Stability research on high water pressure filled karst caves tunnel [Ph.D. thesis], Southwest Jiaotong University, Chengdu, 2009.

[28] Y. Q. Hu, Y. S. Zhao, and D. Yang, "Simulation theory \& method of 3D solid-liquid coupling," Journal of Liaoning Technical University, vol. 2, pp. 204-206, 2007.

[29] S. C. Li, Y. Zhou, and L. P. Li, "Development and application of a new similar material for underground engineering fluid-solid coupling model test," Chinese Journal of Rock Mechanics and Engineering, vol. 31, no. 6, pp. 1128-1137, 2012.

[30] Y. C. Yuan, Study on precursor information evolution law of water inrush and early warming method in tunnels and its engineering applications [Ph.D. thesis], Shandong University, Jinan, 2017.

[31] S. G. Song, S. C. Li, L. P. Li et al., "Model test study on vibration blasting of large cross-section tunnel with small clearance in horizontal stratified surrounding rock," Tunnelling and Underground Space Technology, vol. 92, p. 103013, 2019.

[32] L. P. Li, C. S. Shang, K. W. Chu et al., "Large-scale geomechanical model tests for stability assessment of super-large cross-section tunnel," Tunnelling and Underground Space Technology, vol. 109, article 103756, 2021.

[33] Z. P. Meng, M. S. Li, and P. Q. Lu, "Temperature and pressure under deep conditions and their influences on mechanical properties of sandstone," Chinese Journal of Rock Mechanics and Engineering, vol. 25, no. 6, pp. 1177-1181, 2006.

[34] H. R. Li, C. H. Yang, and Y. G. Liu, "Experimental research on ultrasonic velocity and acoustic emission properties of granite under failure process," Chinese Journal of Geotechnical Engineering, vol. 36, no. 10, pp. 1915-1923, 2014.

[35] J. Li, J. H. Yue, Y. Yang, X. Zhan, and L. Zhao, "Multi-resolution feature fusion model for coal rock burst hazard recognition based on acoustic emission data," Measurement, vol. 100, pp. 329-336, 2017.

[36] X. P. Zhang, Q. Zhang, and S. C. Wu, "Acoustic emission characteristics of the rock-like material containing a single flaw under different compressive loading rates," Computers and Geotechnics, vol. 83, pp. 83-97, 2017.

[37] S. C. Li, X. J. Xu, and Z. Y. Liu, "Electrical resistivity and acoustic emission response characteristics and damage evolution of sandstone during whole process of uniaxal compression," Chinese Journal of Rock Mechanics and Engineering, vol. 1, no. 33, pp. 14-23, 2014. 
[38] S. C. Li, L. P. Li, S. C. Li et al., "Development and Application of Similar Physical Model Test System for Water Inrush of Underground Engineering," Journal of Mining \& Safety Engineering, vol. 27, no. 3, pp. 299-304, 2010.

[39] S. C. Li, C. Hu, L. P. Li, S. G. Song, Y. Zhou, and S. S. Shi, "Bidirectional construction process mechanics for tunnels in dipping layered formation," Tunnelling and Underground Space Technology, vol. 36, pp. 57-65, 2013.

[40] S. X. Ling, Y. Ren, and X. Y. Wu, "Study on reservoir and water inrush characteristic in Nibashan tunnel, Sichuan Province, China," Engineering Geology for Society and Territory, vol. 6, pp. 577-582, 2015.

[41] Z. H. Wang, T. Su, H. Konietzky, Y. L. Tan, and G. L. Zhou, "Hydraulic properties of Beishan granite after different high temperature treatments," Bulletin of Engineering Geology and the Environment, vol. 80, no. 4, pp. 2911-2923, 2021.

[42] G. M. Zhang, K. Zhang, L. J. Wang, and Y. Wu, "Mechanism of water inrush and quicksand movement induced by a borehole and measures for prevention and remediation," Bulletin of Engineering Geology and the Environment, vol. 74, no. 4, pp. 1395-1405, 2015.

[43] H. Yuan, H. M. Zhong, and D. J. Lai, "Geological disaster online realtime monitoring and early-warning system research based on the Flex Viewer framework," Journal of Natural Disasters, vol. 22, no. 2, pp. 177-184, 2013.

[44] S. E. Ma, Z. R. Mei, and J. W. Zhang, "Geological structure quantitative evaluation and monitoring and early warning of water inrush and mud disaster in long tunnel," Modern Tunnel Technology, vol. 46, no. 2, pp. 99-104, 2009.

[45] Y. Q. Xu and J. Ma, "Dynamic monitoring and early warning system for geological disasters based on internet of things technology and its architecture," Chinese Journal of Geological Hazard and Control, vol. 24, no. 3, pp. 90-93, 2013.

[46] G. L. Sun, Z. G. Tao, and W. L. Gong, "Slope disaster monitoring and early warning network system and its engineering application," Journal of China University of Mining and Technology, vol. 46, no. 2, pp. 285-291, 2017.

[47] J. P. Sun, "Technologies of monitoring and communication in the coal mine," Journal of China Coal Societ, vol. 35, no. 11, pp. 1925-1929, 2010.

[48] S. Wang, L. P. Li, S. Cheng et al., "Study on an improved realtime monitoring and fusion prewarning method for water inrush in tunnels," Tunnelling and Underground Space Technology, vol. 112, article 103884, 2021.

[49] X. Kuang, M. Z. Bai, and C. L. Wang, "Research of comprehensive warning of water inrush hazards in karst tunnel based on fuzzy evolution method," Journal of Highway and Transportation and Development, vol. 27, no. 11, pp. 100-103, 2010.

[50] P. Zhao, X. L. Pei, and J. Xue, "Research on safety pre-warning management of building construction based on information fusion technique," China Safety Science Journal, vol. 19, no. 10, pp. 106-110, 2009.

[51] Y. N. Yang, H. P. Cao, and M. Xu, "Index system of risk assessment on karst tunnel water inrush disasters and value of quantitative method," Geosience, vol. 2, pp. 414-420, 2015.

[52] J. Liu and W. M. Wu, "Research on safety risk early warning model of large coal water disaster," Chinese Coal, vol. 5, pp. 122-124, 2017.

[53] P. Liu, J. F. Wang, and L. Y. Zhang, "Reliability study on coal mine water disaster early warning system based on extension evaluation," Coal Technology, vol. 34, no. 6, pp. 136-138, 2015.
[54] S. C. Li, Z. Q. Zhou, L. P. Li, Z. H. Xu, Q. Q. Zhang, and S. S. Shi, "Risk assessment of water inrush in karst tunnels based on attribute synthetic evaluation system," Tunnelling and Underground Space Technology, vol. 38, pp. 50-58, 2013.

[55] L. P. Li, S. C. Li, and Q. S. Zhang, "Study of mechanism of water inrush induced by hydraulic fracturing in karst tunnels," Rock and Soil Mechanics, vol. 31, no. 2, pp. 523-528, 2010.

[56] S. C. Li, Y. C. Yuan, and L. P. Li, "Water inrush mechanism and minimum safe thickness of rock wall of karst tunnel face under blast excavation," Chinese Journal of Geotechnical Engineering, vol. 37, no. 2, pp. 313-320, 2015.

[57] Z. Q. Zhou, Evolutionary mechanism of water inrush through filling structures in tunnels and engineering applications [Ph.D. thesis], Shandong University, Jinan, 2016.

[58] S. J. Zang, "Theoretical on study minimum safe thickness of floors of tunnels in heavy karst areas during construction," Tunnel Construction, vol. 27, no. 5, pp. 17-19, 2007.

[59] M. Sun and W. N. Liu, "Research on water inrush mechanism induced by karst tunnel face with high risk," Rock and Soil Mechanics, vol. 32, no. 4, pp. 1175-1180, 2011.

[60] S. Cheng, Microseismic mechanism and monitoring analysis method for water inrush in tunnels [Ph.D. thesis], Jinan, Shandong University, 2019.

[61] B. Liu, Research on real-time monitoring of tunnel waterbearing geological structure based on resistivity method and IP method for advanced detection and water inrush disaster [Ph.D. thesis], Shandong University, Jinan, 2010.

[62] R. S. Jian, X. H. Ya, and H. M. Sun, "Situation assessment method of rock burst based on multi-source information fusion," Journal of Mining \& Safety Engineering, vol. 31, no. 2, pp. 187-195, 2014.

[63] S. Bjørn and S. W. Hystad, "Situation awareness as a determinant for unsafe actions and subjective risk assessment on offshore attendant vessels," Safety Science, vol. 93, pp. 214-221, 2017.

[64] Y. Wei, Y. F. Lian, and D. G. Feng, “A network security situational awareness model based on information fusion," Journal of Computer Research and Development, vol. 46, no. 3, pp. 353-362, 2009.

[65] Y. Wei and Y. F. Lian, "A network security situational awareness model based on log audit and performance correction," Chinese Journal of Computers, vol. 32, no. 4, pp. 763-772, 2009.

[66] S. Wang, Regional dynamic risk assessment and early warning of tunnel water inrush and application [M.S. thesis], Shandong University, Jinan, 2016. 